\title{
A Novel Approach to Estimate the Radius Ratio of an Elastic Tube from Its Form Function Using Soft Computing Techniques
}

\author{
Younes Khandouch ${ }^{1 *}$, El Houcein Aassif ${ }^{1}$, Said Agounad ${ }^{1}$, Gérard Maze ${ }^{2}$ \\ ${ }^{1}$ LMTI, Laboratoire de Métrologie et Traitement de l'Information, Faculté des Sciences, Université Ibn Zohr, B.P \\ 8106 Agadir, Maroc. \\ ${ }^{2}$ LOMC, Laboratoire Ondes et Milieux Complexes UMR CNRS 6264, Université du Havre, 75 rue Bellot, CS 80 \\ 540, 76085 Le Havre, France. \\ * Corresponding author’s Email: khandouch.younes@edu.uiz.ac.ma
}

\begin{abstract}
A number of researches in different areas has been conducted on using the Principal Component Analysis PCA combined with Artificial Neural Networks ANN. In this current research, we use these two techniques to estimate the radius ratio b/a (a: outer radius, b: inner radius) of an elastic tube based on its form function. The PCA technique is used to estimate the component loadings corresponding to form function. Then, the component loadings it's used in the ANN technique to estimate the radius ratio of the tube. To get the optimal network, several configurations are implemented and tested. The optimal configuration selected is a network with 16 inputs, 2 hidden layers composed of 4 and 1 neurons respectively, and trained by the back-propagation algorithm. This configuration is able to estimate the radius ratio $b / a$ with a mean absolute error MAE of about 0.0024 and a mean square error MSE of 0.0008 . This study reveals benefits of the combination between PCA and ANN, and also it provides some new ideas for further researches. This current work can be used as a novel approach for the characterization of an elastic tube.
\end{abstract}

Keywords: Radius ratio, estimation, form function, principal component analysis, artificial neural networks.

\section{Introduction}

Currently, soft computing techniques which differs from the traditional methods has been widely used to solve complex nonlinear problems by applying different approaches [1-4]. Recently there has been an increasing interest for the use of ANN approach [5-6]. It's a computational model inspired from natural biological neural networks. It consisting of a large number of interconnected processing neurons that simulates the human brain learning [6]. The ANN allows to learn easily to estimate relationships between one or several input variables called independent variables and one or several output variables called dependents variables without a specific mathematical function. Hence, an ANN works properly for solving complicated non-linear problems of multivariate systems. In addition, many input variables may cause poor generalization performance [7]. These problems can be solved by combining artificial neural network with principal component analysis [8-9]. PCA transforms the original data set into a set of uncorrelated variables that capture all of the variance of the original data set [10]. On the other hand, if the database is limited and contains qualitative information, the combined system can be used in modelling complex system behaviors [11].

The aim of the present study is to investigate and to explore the capability of combining both approaches of Neural Networks and Principal Component Analysis to estimate the radius ratio $b / a$ (a: outer radius, b: inner radius) based on backscattering form function by an immersed elastic tube. In previous works, many theoretical and experimental studies show that acoustic resonances of a cylindrical shell are related to its physical and geometrical proprieties [12-17]. Conversely, starting from the resonances of circumferential wave, we can 
characterize material constituting a cylindrical shell and its geometry [18]. This study use a close relationship between circumferential waves propagates in the case of an elastic tube, and lamb waves propagates in the case of a plat at same thickness. The necessary condition is to check this similarity between these two types of waves. It must be used for an elastic tube (radius ratio $b / a>0.90$ ) and a plat of same thickness $(e=a-b)$. The similarity allows to use the traditional relationships applied in the case of Lamb waves to go up to the value of the reduced cut-off frequency $(k a) c$ in the case of a tube. The result obtain by this study is limited to an elastic tube with radius ratio $(b / a>$ $0.90)$. In this term paper we explore the capability to estimate the radius ratio $b / a$ of an elastic tube with radius ratio $(b / a>0.50)$ to resolve this problem. We introduce a new approach which is based on soft computing technique to develop a model that is capable to estimate the radius ratio of an elastic tube using backscattering form function. This problem is divided into two main phases: the first is the preprocessing and component loadings estimation phase. The second is the estimation phase, where a number of trial is tested until we carried out less errors to find the best network architecture. To perform, many configurations are evaluated and tested.

In this work, the approach based on soft computing techniques was proposed. The PCA is used to estimate the component loadings corresponding to form function, and a neural networks model was developed and expanded with input parameters which are the component loadings estimated by the PCA technique in order to return the radius ratio corresponding to form function. The combined method based on PCA and ANN usually improves the training speed, as well as it enhance the robustness of the model and reduces model errors. The capability to estimate the radius ratio of an elastic tube using the proposed approach was tested. This approach does not present any approximation as in the case of the natural modes method which assimilates the tubes to the plates with the same thickness. This approximation is valuable for a thin tubes with radius ratio $b / a>0.9$. In the despite, the introduced approach is able to generalize the solution to a thick tubes, so whatever the value of their radius ratio.

\section{Backscattering response from an elastic tube}

If an air-filled elastic tube with radius ratio $b / a$ immersed in water and excited by a plane acoustic wave perpendicularly to its axis, the shell and the water-shell interface automatically generate

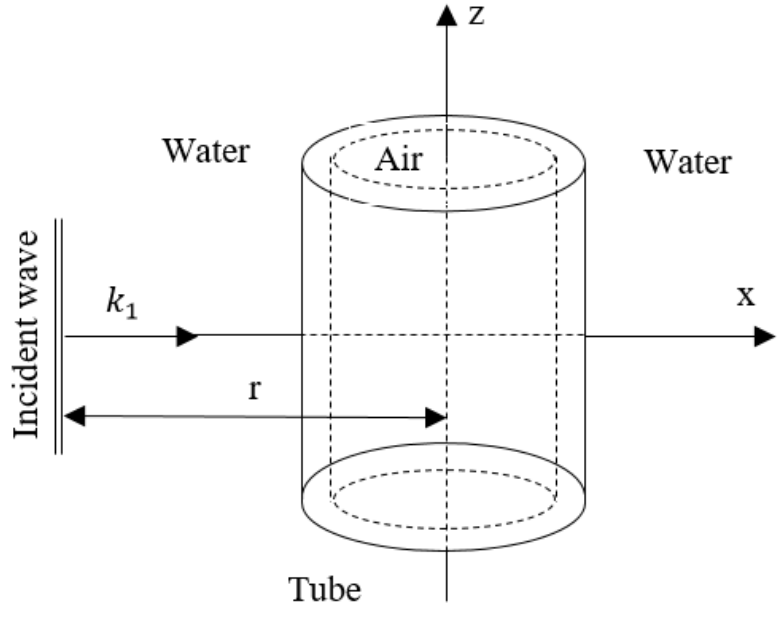

Figure. 1 The geometry used for formulating the backscattered complex pressure by an elastic tube.

circumferential waves. The results shows two types of circumferential waves: the symmetric waves and the anti-symmetric waves [20-23]. Fig. 1 shows the cylindrical coordinate orientation and the direction of the incident plane wave [24-28]. These circumferential waves make standing waves on the circumference of the tube constituting resonances. For a tube made in a given material, the resonance dimensionless frequencies of these waves essentially depend on the radius ratio b/a. These resonances are observed on the spectrum of the acoustic pressure backscattered by the tube [25, 28-30]. The mode $n$ of each resonance is the number of wavelengths around the circumference.

The complex pressure Pscat (form function) backscattering by a tube is the summation of the modes that takes into account the effects of the incident wave, the reflective wave $\{1\}$, circumferential waves in the shell $\{2\}$ (whispering gallery waves, Rayleigh wave), and interface Scholte wave $\{3\}$, connected to the geometry of the object Fig. 2.

To explain these effects, it is necessary to apply the Sommerfeld-Watson transform [17, 31]. The

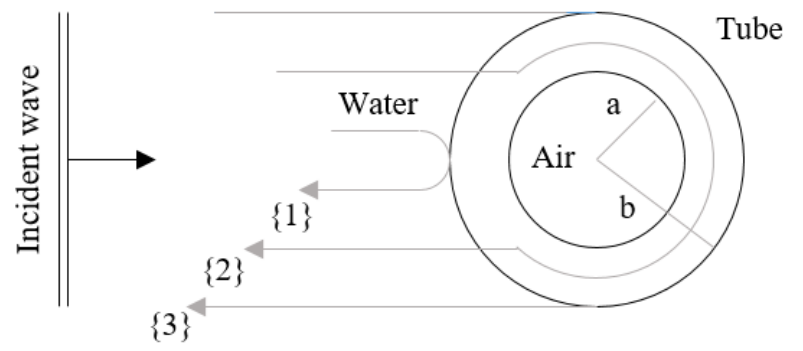

Figure. 2 Mechanisms of the formation of echoes $\{1\}$, specular reflection; $\{2\}$, circumferential shell, and $\{3\}$, Scholte waves and Franz waves. 
general form of the scattered pressure field in a plan perpendicular to the $\mathrm{z}$-axis can be expressed as [3235]:

$$
\begin{aligned}
& P_{\text {scat }}(\omega) \\
& =P_{0} \frac{1-i}{\sqrt{\pi k_{1} r}} \exp i\left(2 k_{1} a\right) \sum_{n=0}^{\infty} \varepsilon_{n} \frac{D_{n}^{1}(\omega)}{D_{n}(\omega)} \cos (n \theta)
\end{aligned}
$$

where ( $\omega$ : angular frequency, $k_{1}=\omega / C_{1}$ : wave number with $C_{1}$ is the wave velocity in the external fluid, $P_{0}$ : amplitude of the incident plane wave, $D_{n}^{1}(\omega)$ and $D_{n}(\omega)$ : determinants computed from the boundary conditions of the problem on the two interfaces, $\varepsilon_{n}$ : Neumann coefficient $\left(\varepsilon_{n}=1\right.$ if $n=$ 0 and $\varepsilon_{n}=2$ if $n \neq 0$ ), $r$ : distance between the position where the pressure is calculated and the $z$ axis of the tube.

The complex backscattering pressure computed in a far field is obtained for $\theta=\pi$ as a function of the dimensionless frequency $x_{1}$ given by the relation:

$$
x_{1}=k_{1} a=\frac{2 \pi v a}{C_{1}}
$$

where $v$ is the wave frequency in hertz.

The physical parameters used in the calculation of the backscattering complex pressure are illustrated in Table 1. Fig. 3 show the module of the backscattered complex pressure calculated for tree
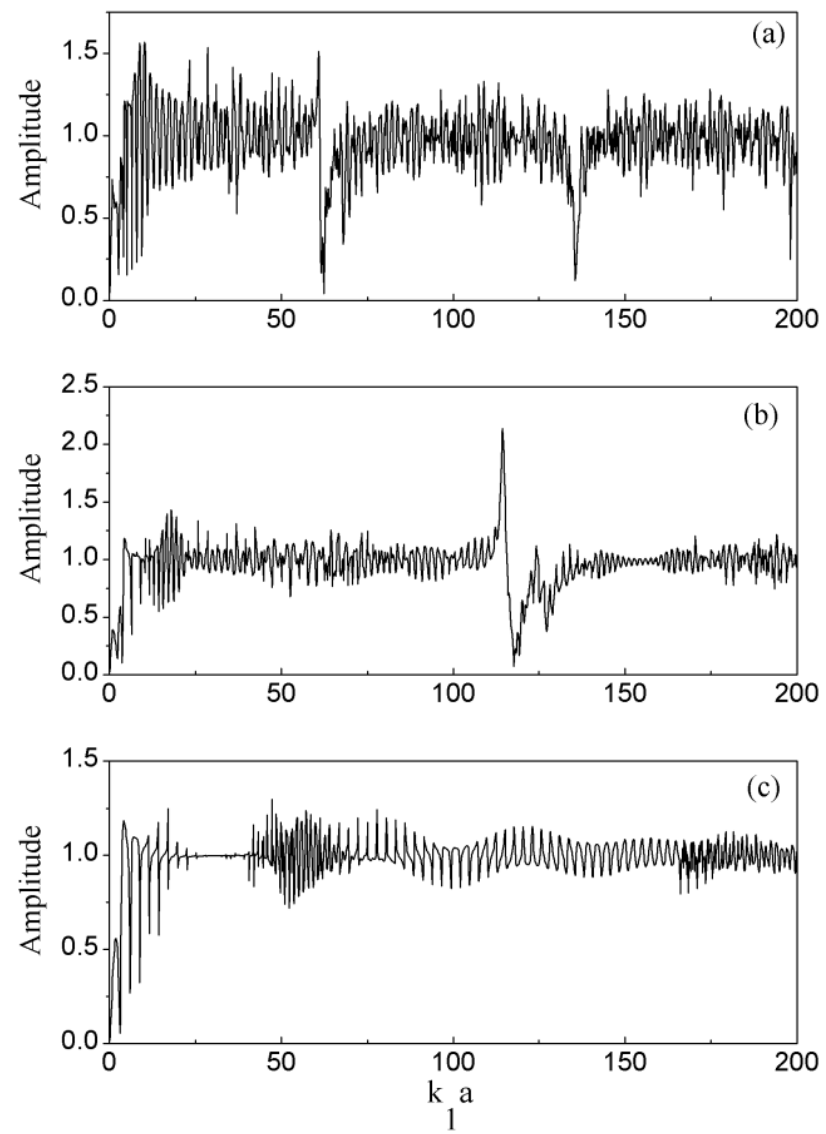

Figure. 3 Backscattered pressure spectrum by a copper tube with radius ratio: $(a) b / a=0.85$, (b) $b / a=$ $0.92,(c) b / a=0.98$
Table 1. Physical parameter.

\begin{tabular}{|c|c|c|c|}
\hline & $\begin{array}{c}\text { Density } \\
\boldsymbol{\rho}\left(\boldsymbol{K g} / \boldsymbol{m}^{\mathbf{3}}\right)\end{array}$ & $\begin{array}{c}\text { Longitudinal } \\
\text { velocity } \\
\boldsymbol{C}_{\boldsymbol{l}}(\boldsymbol{m} / \boldsymbol{s})\end{array}$ & $\begin{array}{c}\text { Transvers } \\
\text { velocity } \\
\boldsymbol{C}_{\boldsymbol{t}}(\boldsymbol{m} / \boldsymbol{s})\end{array}$ \\
\hline Cupper & 8920 & 4760 & 2325 \\
Water & 1000 & 1470 & - \\
Air & 1.29 & 334 & - \\
\hline
\end{tabular}

examples of copper tube with radios ratio ((a) $b / a=$ 0.85 , (b) $b / a=0.92$ and (c) $b / a=0.97$ ) in function of the reduced frequency $K a$. The backscattered pressure are presented respectively.

\section{Principal component analysis}

The principal Component Analysis technique is one of multivariate data analysis methods $[10,36]$. It has been widely used by almost all scientific disciplines. It was first introduced by Karl Pearson [37]. Over twenty years later, Fisherand Mckenzie proposed the first algorithm to PCA which is known as a NIPALS [38]. However, Hotlling made the major developmental impact on the method [39]. The central idea of this method is to reduce the number of study variables with retaining the much information as possible. This reduction is achieved by transforming the given variables of study to a new set of variables whose are independents and linear compound of input variables.

Mathematically, PCA is an orthogonal transformation technique of the given variables which its goal is to form a new variables that are uncorrelated by taking linear combinations of the original variables. The new variables are called principal components [40]. The maximum variance of the original data are represented by the first principal component, the second one defines the next greatest variation not explained by the first component. Then, the third explains as much variation not extracted by the first and the second component, and so forth. In practice, for modeling a multivariate data using the PCA technique, the data are collected in a matrix. In order to make the result independent of the used units for each variable, the data matrix are normalized in order to use it in the calculation of the correlation matrix defined by:

$$
\mathrm{C}_{x x}=\frac{1}{N-1} X^{T} X
$$

$\mathrm{N}$ : Number of observations of each variable and $\mathrm{X}$ : normalized data matrix.

The calculation of PCA parameters can be summarized in the calculus of eigenvalues and eigenvectors of the correlation matrix $\mathrm{C}_{x x}$. From the spectral decomposition, this matrix can be written as follows: 


$$
\mathrm{C}_{x x}=P \Lambda P^{T}=\sum_{i=1}^{M} \lambda_{i} p_{i} p_{i}^{T}
$$

Where, $p_{i}$ is the $i^{\text {th }}$ eigenvector of $\mathrm{C}_{x x}, \lambda_{i}$ is the corresponding eigenvalue and $\mathrm{M}$ number of variables.

If there is one or more linear relationships between input data, we have one or more eigenvalues equal to zero and the matrix $X$ can be represented by the first components corresponding to eigenvalues not equal to zero. However, the eigenvalues equal zero are rarely come across in practice. So, it is necessary to determine the number which represents the number of components corresponding to the dominant eigenvalues. Many rules are proposed in the literature to determine the number of components to retain [41]:

- Criterion of sufficient quality of representation: it's the measure the percent variance explain by the first components, and retain all component needed to explain the desired one.

- Kaiser criterion [42]: is to retain any components with eigenvalues great than 1.0 Components that have a substantial contribution to original data.

- Cattell criterion [43]: also called the scree test, it's a graphical method performed by plotting the eigenvalues against their respective component number, and retaining the number of components that come before a break in the plot.

It appears that the Kaiser criterion sometimes keep too many components than the Cattell criterion [41]. Both criterion do perfectly in normal conditions. In practice, an important aspect has been provided so that the solution must be interpretable. Therefore, on examines several solutions with small or high number of components, and chooses the one that makes the best sense, so like in this research.

\section{Artificial neural networks}

The artificial neural networks technique is an alternate computational approach inspired by studies of brain and nervous systems [5-6]. It is based on theories of the massive interconnection and parallel processing architecture of biological neural systems. Indeed, the advantage of neural networks is that they are capable of modelling non-linear systems. Currently many researchers applied neural networks model in estimation study.

Back-Propagation neural network [44] that is characterized by the error backpropagation the last to the first layer, is the best and the most popular multilayer neural network [45]. In practice, BP neural network consisting of three adjacent layers, input, hidden and output layers, the input one consisting of neuron representing various input variables. The hidden layers consist of many hidden neuron and an output layer consisting of output variables. Each neuron in a certain layer is connected to every single neuron in the next layer by links having an appropriate and an adjustable connection weight. The input neuron pass on the input signal values to the neuron in the hidden layers. The values are distributed to all the neuron in the hidden layers depending on the connection weights between the input neuron and the hidden neurons. Fig. 4 shows corresponding architecture of the neural network used in this study and the schematic representation of a neuron.

The training of neural networks is as follows: for the neuron $j$, with input $x_{i}$, its output $O_{j}$ is calculated with the following formula:

$$
o_{j}=f\left(y_{j}+b_{j}\right) \quad ; \quad y_{j}=\sum_{i=1}^{n} \omega_{i j} x_{i}
$$

where $\omega_{i j}$ is the connection weights between the neurons in the previous layer and the actual neurons, $b_{j}$ is the bias input of the actual neurons, $\mathrm{n}$ is the number of input, and $f$ is the activation function of the neurons is usually a sigmoid function defined by:

$$
f(x)=\frac{1}{1+e^{-x}}
$$

The back-propagation algorithm is used to minimize the error of the output defined as:

$$
E=\frac{1}{2} \sum_{p=1}^{l} \sum_{k=1}^{m}\left(T_{p k}-O_{p k}\right)^{2}
$$

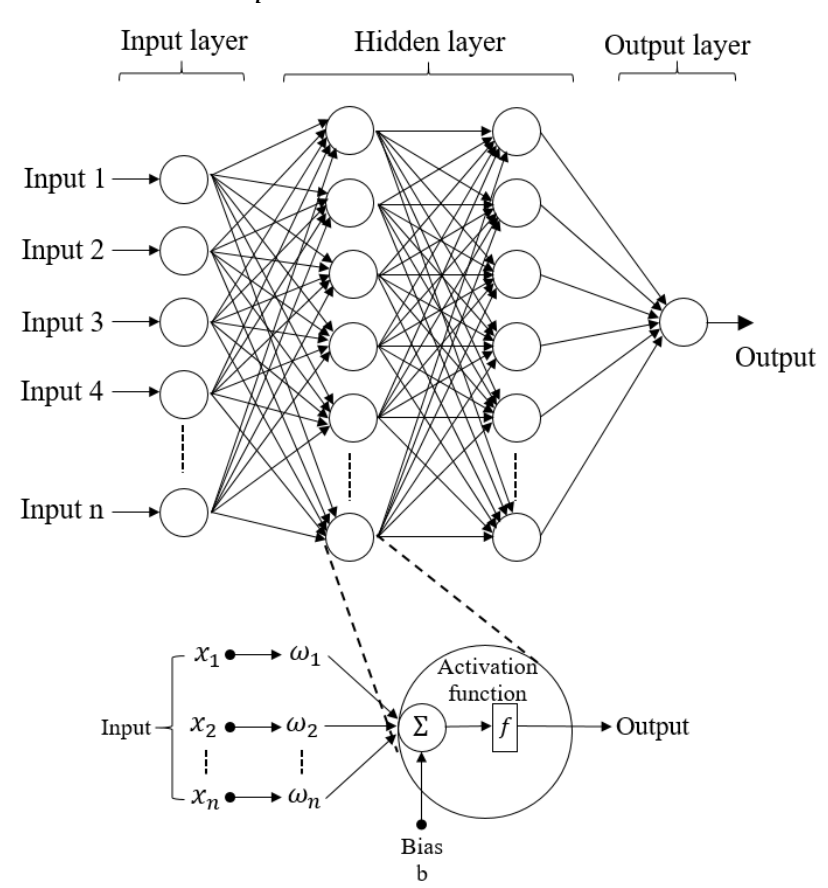

Figure. 4 Neural networks architecture and the schematic representation of a neuron. 
where $l$ is the number of training example, $m$ is the number of output neurons, $T_{p k}$ is the target output of $k^{\text {th }}$ neuron of $p^{\text {th }}$ training example, and $O_{p k}$ is the output value of $k^{\text {th }}$ neuron of $p^{\text {th }}$ training example. In the training process, when the error falls below the threshold or tolerance level, the training will stop. The error $\delta_{k}$ in output layer and error $\delta_{j}$ in hidden layer are defined as:

$$
\begin{gathered}
\delta_{k}=\lambda\left(T_{k}-O_{k}\right) f^{\prime}\left(O_{k}\right) ; \\
\delta_{j}=\lambda \sum_{i=1}^{n} \delta_{l} \omega_{i j} x_{i} f^{\prime}\left(O_{j}\right)
\end{gathered}
$$

where $T_{k}$ is the target output and $O_{k}$ is the actual output of the $k^{\text {th }}$ output neuron in the output layer, $O_{j}$ is the actual output value in the hidden layer, and $\lambda$ is the adjustable variable in the activation function. In the training process the weights and biases in both output and hidden layers are modified repeatedly, there new values are written as:

$$
\begin{gathered}
\omega_{i j}(k+1)=\omega_{i j}(k)+\eta \delta_{j} O_{i} \\
b_{j}(k+1)=b_{j}(k)+\eta \delta_{i}
\end{gathered}
$$

where $k$ is the number of the epoch and $\eta$ is the learning rate.

This modification proceeds downward. Through such an iterative process, the network attains the ability to promptly output the similar output value to the target output. The performance of the networks depends mainly on data representation [46].

\section{Methodology}

This section describes the steps taken to implement the PCA and the ANN approaches Fig. 5 describe the methodology used in this study.

The first phase consist on the estimation of the component loadings with PCA in two steps. In the first step, we collecting all data base in the input matrix. Then this data is normalized. After that, the PCA technique well applying to the training and validation data set. The principal components was estimated and classified according to their variations. In the second step, the component loadings of the testing data base are estimated. The component loadings is the weighting factors (components coordinates) of principal components for the input data. It's the projection of analyzed data in the space defined by the principal components. This parameter inform how much of the variability of analyzed data is represented by the principal components.

The second phase is implementing the component loadings in the neural networks architectures. For all architectures, the activation function is a differentiable sigmoid function, which helps to apply the non-linear mapping from inputs to outputs, the synaptic weights are initialized randomly. The number of hidden layers vary from 1 to 2 and varying at the same time the number of neurons in hidden layers. The number of neurons in hidden layers can vary according to the complexity of the problem and the size of the data set. The implementation of the neural networks requires [47]: A collection of the data for the training, validation and testing. The training data is the component loadings corresponding to form function in the training data set, it's used to adjust the neural network weights. The validation data set is the component loadings corresponding to form function in the validation data set, it's used to minimize over fitting. No adjustment of the neural network weights occurs with this data set. But, any increase in accuracy over the training data set affect the increase in accuracy over a data set that has not been shown to the network before. If the accuracy over the training data increases, while the accuracy over the validation data stays unchanging or decreases. Then, the network configuration is being over fitted and therefore the training will be stopped. The testing data set is the component loadings corresponding to form function

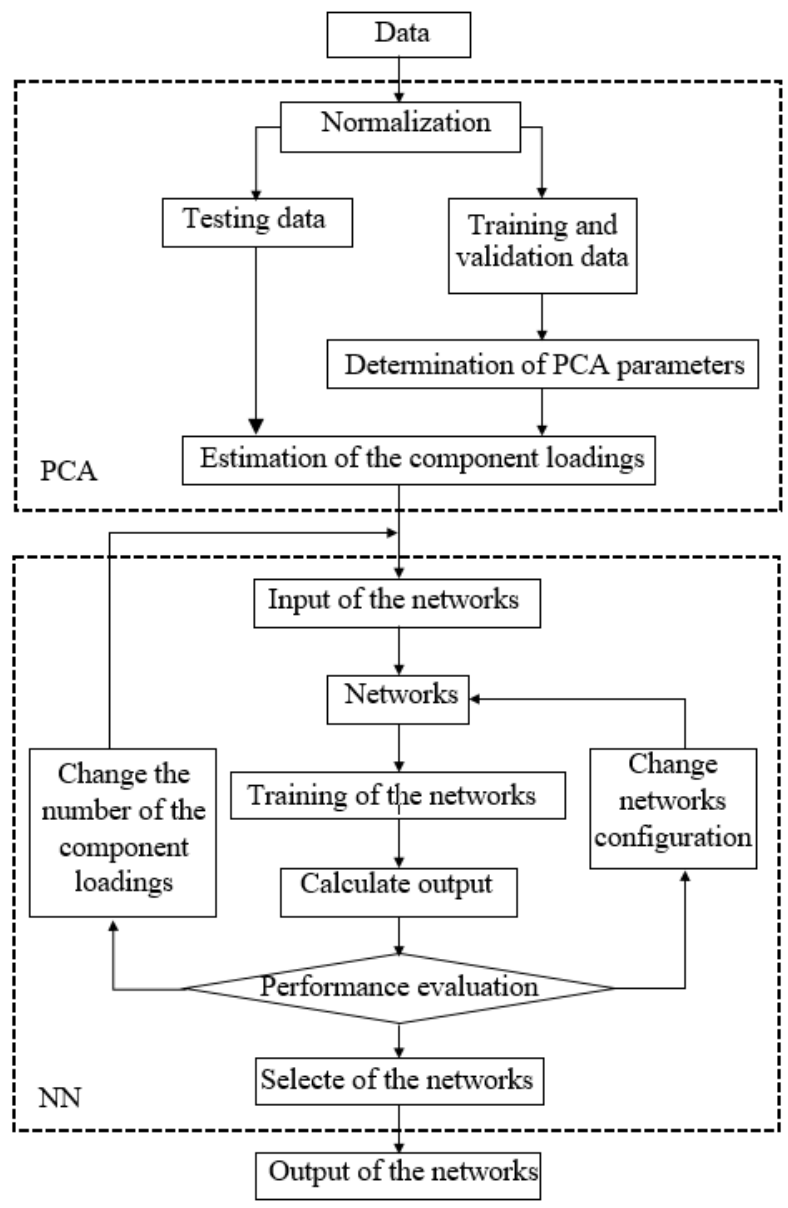

Figure. 5 Methodology of implementation of PCA and ANN 
in the testing data set. It's used for testing the final solution in order to confirm the capability of the learned neural network in estimation.

Then, the output results obtained for each model were compared with the corresponding actual results. ANN model estimation performance can be assessed by statistical coefficients, the mean absolute error MAE, the mean square error MSE, and the correlation coefficient $R$. The corresponding definitions are given as follows:

$$
\begin{gathered}
M A E=\frac{1}{n} \sum_{i=1}^{n}\left|d_{i}-e_{i}\right| \\
M S E=\sqrt{\frac{1}{n} \sum_{i=1}^{n}\left|d_{i}-e_{i}\right|} \\
R=\frac{\sum_{i=1}^{n}\left(d_{i}-\bar{d}\right)\left(e_{i}-\bar{e}\right)}{\sqrt{\sum_{i=1}^{n}\left(d_{i}-\bar{d}\right)^{2} \sum_{i=1}^{n}\left(e_{i}-\bar{e}\right)^{2}}}
\end{gathered}
$$

where $d$ is the desired value, $e$ is the estimated value, $\bar{d}$ is the mean of the desired value, $\bar{e}$ is the mean of the estimated value, and $n$ is the total number of the data. The smaller MAE and MSE values and the larger value of $\mathrm{R}$ represent the less deviation and corresponding to the better performance of the model.

\section{Results and discussion}

The total data set consisted of 50 form functions of different radius ratio from 0.50 to 0.99 . The data is divided into three subsamples: training set $(60 \%)$, validation set $(20 \%)$ and testing set $(20 \%)$. The proportions of the training and the validation sets are selected manually from the data base. After applying PCA to training set and validation set, the PCA parameters was estimated. Fig. 6 shows a

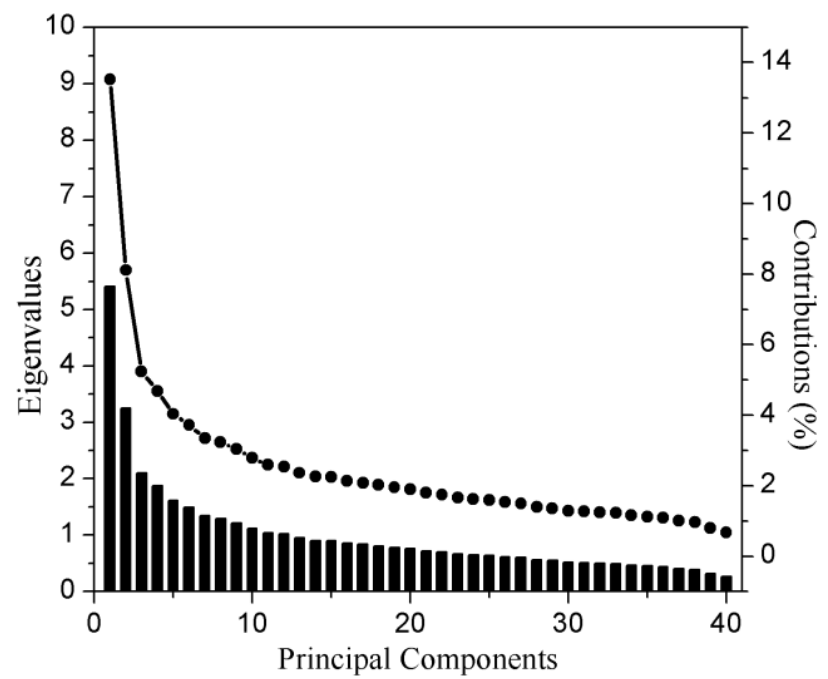

Figure. 6 Eigenvalues and contributions of component to the total variance in terms of principal components.

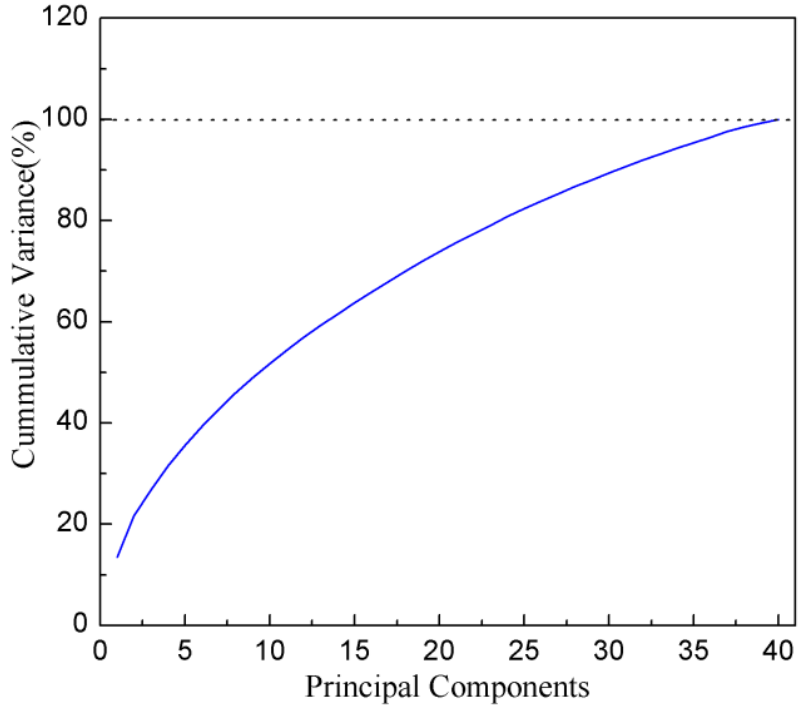

Figure. 7 Representation of cumulative variance in terms of principal components.

representation of eigenvalues in terms of principal components for the models and Fig. 7 shows the relative contribution of each component to the total variance of data. For example, in Fig. 6 the first component is about $13.5 \%$ of the variance; it means that this component represents a significant part on all form function. The components two and three are respectively about $8.1 \%$ and $5.3 \%$ of the variance. The principal components obtained revealed that 12 components with eigenvalue greater than 1 . The components together explain $56.90 \%$ of total variance Fig. 7.

With $n$ original variables, we will obtain $n$ principal components, still have as many new components as original variables except uncorrelated. Often it is desirable to retain a smaller set of the principal components for easier interpretation of the analysis. Then, the component loadings are estimated. So every function form was described by a number of the component loadings correspond to the number of principal components chosen, which is the input of the neural networks architectures developed and trained by the back-propagation algorithm. The reason to train more models is to get the best neural

Table 2. Parameters of different neural networks architecture.

\begin{tabular}{|c|c|c|c|}
\hline & $\begin{array}{c}\text { Number of } \\
\text { input } \\
\text { variables }\end{array}$ & $\begin{array}{c}\text { Number of } \\
\text { hidden } \\
\text { layers }\end{array}$ & $\begin{array}{c}\text { Number of } \\
\text { neurons in } \\
\text { hidden layers }\end{array}$ \\
\hline 1 & 12 & 1 & 9 \\
2 & 16 & 1 & 6 \\
3 & 17 & 1 & 12 \\
4 & 32 & 1 & 1 \\
5 & 10 & 2 & $6 / 10$ \\
6 & 16 & 2 & $4 / 1$ \\
7 & 22 & 2 & $3 / 2$ \\
\hline
\end{tabular}

DOI: $10.22266 /$ ijies2016.0930.14 


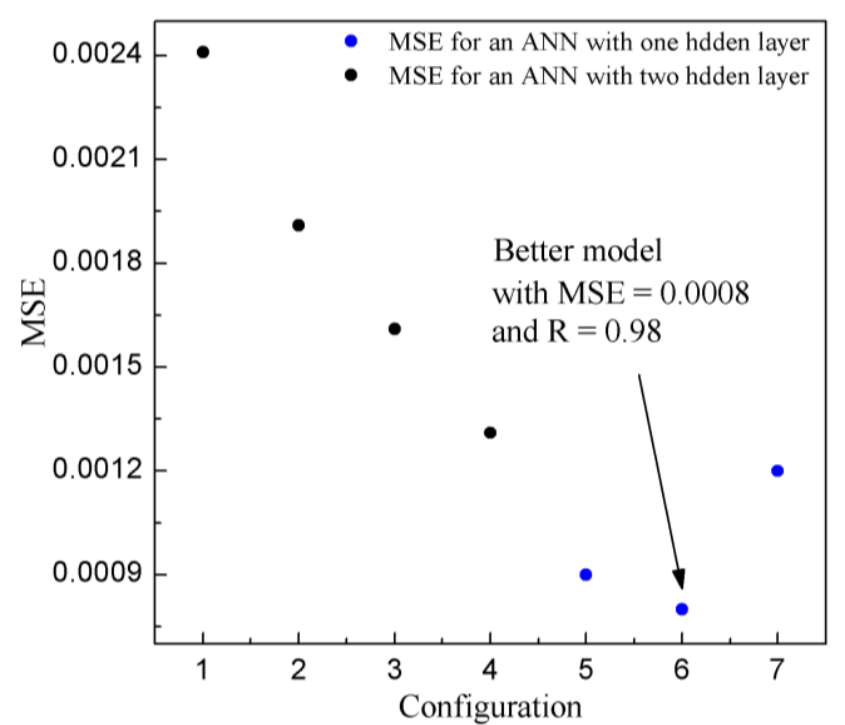

Figure. 8 Errors for the estimation of the radius ratio with different ANN configurations

networks architecture that has the optimum number of inputs, the optimal number of hidden layers and the optimal number of neurons in hidden layers. Table 2 shows the most successful network tested.

The optimal configuration is obtained by the minimization of the difference between the desired and the estimated outputs. Selection of the network was performed at minimum of mean absolute error (MSE). The result of calculated errors are presented in fig. 8. The best architecture is a network with 16 inputs, 2 hidden layers. Each layer is composed of 4 and 1 neurons respectively and 1 neuron in the output layer. The table 3 presents the radius ratio $b / a$ estimated and desired for the testing data set.

The PCA-ANN model output are very similar to desired output. The performance of the model was done according to the mean square error is $M S E=$ 0.0008 and the mean absolute error is $M A E=$ 0.0248 . Fig. 9 shows the estimated values of the radius ratio traced according to the desired values of the radius ratio for testing data. The best results are obtained when the points are illustrated at the straight line. This means that the value of the coefficient of determination $\mathrm{R}$ is 1 . The values of the coefficient of

Table 3 - Values of desired and estimated radius ratio

\begin{tabular}{|c|c|}
\hline Desired radius ratio & Estimated radius ratio \\
\hline 0.500 & 0.539 \\
0.550 & 0.555 \\
0.600 & 0.636 \\
0.650 & 0.676 \\
0.700 & 0.716 \\
0.750 & 0.756 \\
0.800 & 0.844 \\
0.850 & 0.821 \\
0.900 & 0.896 \\
0.950 & 0.909 \\
\hline
\end{tabular}

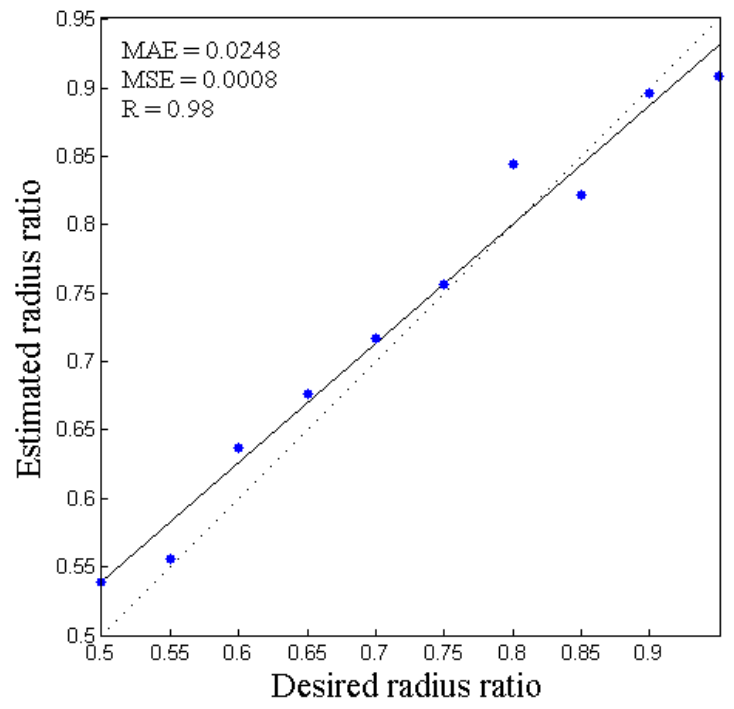

Figure. 9 Correlation of desired and estimated values of radius ratio for testing data

determination $\mathrm{R}$ corresponding to the testing data for the optimal configuration are $R=0.98$. These values show that there is a positive and almost perfect agreement between the desired and estimated radius ratio. Fig. 10 shows the validation performance of the configuration calculated by considering the Mean Squared Error (MSE). As presented in this figure the training of the network was stopped at epoch 18 with a best validation performance equal to 0.0009 .

\section{Conclusion}

The scattering acoustic from elastic tubes is the object of many studies experimental and theoretical. In this study, we have introduced a new approach which is based on soft computing technique to estimate the radius ratio of an elastic tube from its form function. The proposed approach is based on combination between principal component analysis

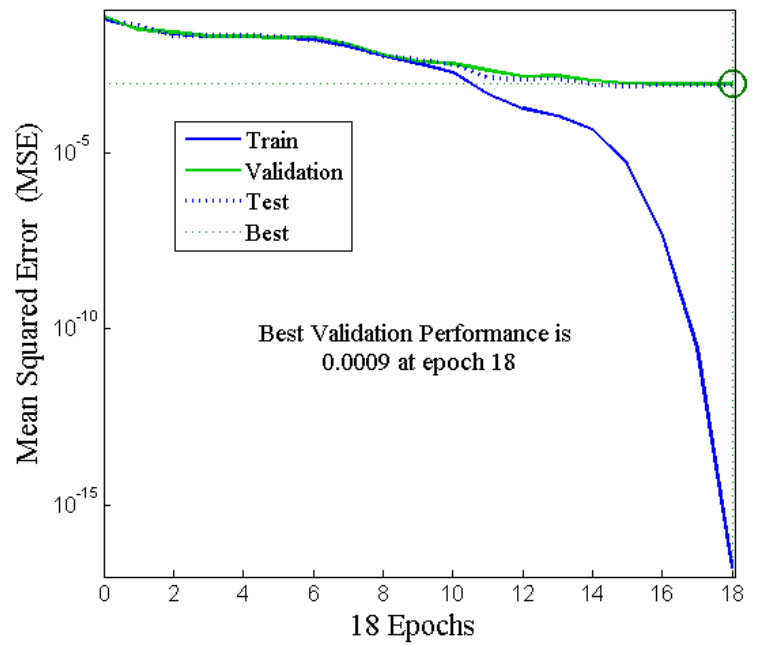

Figure. 10 Visualization of errors of training validation and testing data as a function of the number of iterations. 
and the artificial neural networks techniques. The model which is based on this two techniques has been developed to return the radius ratio corresponding to the form function. In this current research, we have tested the capability to estimate the radius ratio of different tubes made from copper. The introduced model shows almost perfect agreement between desired and estimated radius ratio. This technique can be generalized to other tubes made from different materials and can help us to estimate others parameters related to tube. This study reveals advantages of the application of the proposed approach in scattering acoustic. This research can be used as a new method for the characterization of an elastic tube. In the further researches, with regard to the algorithm, other techniques can be examined to ameliorate this study.

\section{References}

[1] Kumar S. Ray, Soft Computing and Its Applications, Volume One: A Unified Engineering Concept, Apple Academic Press, 2014.

[2] D. Pham, P.T.N. Pham, "Artificial intelligence in engineering", International Journal of Machine Tools and Manufacture. Vol. 39, No.6, pp. 937-949, 1999.

[3] D. Graupe, Principles of artificial neural networks, Vol. 7. World Scientific, 2013.

[4] N. Siddique, H. Adeli. Computational intelligence: synergies of fuzzy logic, neural networks and evolutionary computing. John Wiley \& Sons, 2013.

[5] D. Svozil, V. Kvasnicka, J. Pospichal, "Introduction to multi-layer feed-forward neural networks", Chemometrics and Intelligent Laboratory Systems. Vol. 39, No. 1, pp. 43-62, 1997.

[6] Simon, O. Haykin, Neural Networks and Learning Machines, 3 ed., Upper Saddle River: Pearson, New Jersey, 2009.

[7] S. Walczaka, N. Cerpab, "Heuristic principles for the design of artificial neural networks", Information and Software Technology. Vol. 41, No. 2, pp. 107-117, 1999.

[8] J. Zahedi, M.M. Rounaghi, "Application of artificial neural network models and principal component analysis method in predicting stock prices on tehran stock exchange", Physica A: Statistical Mechanics and its Applications. Vol. 438, pp. 178-187, 2015.

[9] B. Boukhatem, S. Kenai, A. Hamou, D. Ziou, M. Ghrici, "Predicting concrete properties using neural networks (NN) with principal component analysis (PCA) technique", Computers and Concrete. Vol. 10, No. 6, pp. 557-573, 2012.

[10] I.T. Jolliffe, Principal Component Analysis, 2 ed., Springer-Verlag, New York. 2002.

[11] M. Moshgbar, R. Parkin, R.A. Bearman, "Application of fuzzy logic and neural network technologies in cone crusher control”, Minerals Engineering. Vol. 8, No. 1-2, pp. 41-50, 1995.

[12] A. Elhanaoui, E. H. Aassif, G. Maze, D. Décultot, "Acoustic scattering by a two-layer cylindrical tube immersed in a fluid medium: Existence of a pseudo wave", Ultrasonics. Vol. 65, pp. 131-136, 2016.

[13] A. Dariouchy, E. H. Aassif, D. Décultot, and G. Maze, "Acoustic characterization and prediction of the cutoff dimensionless frequency of an elastic tube by neural networks", IEEE Transactions on Ultrasonics, Ferroelectrics, and Frequency Control. Vol. 54, No. 5, pp. 1055-1064, 2007.

[14] R. Latif, E. Aassif, A. Moudden, D. Decultot, B. Faiz, G. Maze, "Determination of the cutoff frequency of an acoustic circumferential wave using a time-frequency analysis", NDT \& EInternational. Vol. 33, No. 6, pp. 373-376, 2000.

[15] L. Haumesser, D. Dcultot, F. Lon, G. Maze, "Experimental identification of finite cylindrical shell vibration modes", Journal of the Acoustical Society of America. Vol. 111, No. 5, pp. 2034-2039, 2002.

[16] J.D.N. Cheeke, X. Li, Z. Wang, "Observation of flexural lamb waves (A0 mode) on water-filled cylindrical shells", Journal of the Acoustical Society of America. Vol. 104, No. 6, pp. 3678-3680, 1998.

[17] P. Marston, N. Sun, "Backscattering near the coincidence frequency of a thin cylindrical shell: Surface wave properties from elastic theory and an approximate ray synthesis", Journal of the Acoustical Society of America. Vol. 97, No. 2, pp. 777-783, 1995.

[18] R. Latif, E. Aassif, M. Laaboubi, G. Maze, "Determination of the thickness of elastic tube using thetime-frequency analysis of wigner-ville", Acta Acustica united with Acustica. Vol. 95, No. 5, pp. 253-257, 2009.

[19] F. Bellamine, A. Elkamel, "Model order reduction using neural network principal component analysis and generalized dimensional analysis", Engineering Computations. Vol. 25, No. 5, pp. 443-463, 2008.

[20] F. Chati, F. Léon, G. Maze, "Acoustic scattering by a metallic tube with a concentric solid polymer cylinder coupled by a thin water layer. Influence of the thickness of the water layer on the two ScholteStoneley waves", Journal of the Acoustical Society of America. Vol. 118, No. 5, pp. 2820-2828. 2005.

[21] S. J. Rupitsch, and J. Ilg, "Complete Characterization of Piezoceramic Materials by Means of Two BlockShaped Test Samples", IEEE Transactions on Ultrasonics, Ferroelectrics, and Frequency Control. Vol. 62, No. 7, pp. 1403-1413, 2015.

[22] L. Haumesser, N. Touraine, D. Décultot, G. Maze, "Acoustic scattering from finite cylindrical shells: Influence of end-caps (L)", Journal of the Acoustical Society of America. Vol. 116, No. 4, pp. 1901-1904, 2004. 
[23] G. Maze, J. Izbicki, J. Ripoche, "Resonances of plates and cylinders: Guided waves", Journal of the Acoustical Society of America. Vol. 77, No. 4, pp. 1352-1357, 1985.

[24] M. Rajabi, M. Behzad, "An exploration in acoustic radiation force experienced by cylindrical shells via resonance scattering theory", Ultrasonics. Vol. 54, No. 4, pp 971-980, 2014.

[25] L. Flax, L. Dragonette, H. berall, "Theory of elastic resonance excitation by sound scattering", Journal of the Acoustical Society of America. Vol. 63, No. 3, pp. 723-731, 1978.

[26] P. Pareige, P. Rembert, J.L. Izbickiand, G. Maze, J. Ripoche, "Méthode impulsionnelle numérique MIN pour l'isolement et l'identification des résonances de tubes immergés", Physics letters. A. Vol. 135, No. 2, pp. 143-146, 1989.

[27] J. Murphy, E. Breitenbach, H. Uberall, "Resonance scattering of acoustic waves from cylindrical shells", Journal of the Acoustical Society of America. Vol. 64, No. 2, pp. 677-683, 1978.

[28] G. Maze, Diffusion d'une onde acoustique plane par des cylindres et tubes immergés dans l'eau: Isolement et identification des résonances, Thèse de Doctorat d'Etats-sciences, University of Rouen, Rouen, France. 1984.

[29] L. Bai, A. Velichko, and B. W. Drinkwater, "Ultrasonic Characterization of Crack-Like Defects Using Scattering Matrix Similarity Metrics", IEEE Transactions on Ultrasonics, Ferroelectrics, and Frequency Control. Vol. 62, No. 3, pp. 545-559, 2015.

[30] M. Rajabi, M. Behzad, Interaction of a plane progressive sound wave with anisotropic cylindrical shells, Composite Structures. Vol. 116, pp. 747-760, 2014.

[31] G. Frisk, J. Dickey, H. Uberall, "Surface wave modes on elastic cylinders", Journal of the Acoustical Society of America. Vol. 58, No. 5, pp. 996-1008, 1975.

[32] J. Van de Loock, D. Décultot, F. Léon, F. Chati, G. Maze, and D. R. Rajaona, "Acoustic radiation of a submerged cylindrical shell in low frequency", Journal of the Acoustical Society of America. Vol. 113, No. 1, 2013.

[33] M. Laaboubi, E.H. Aassif, R. Latif, A. Dliou, G. Maze, D. Décultot, "Application of the reassignment timefrequency method on an acoustic signals backscattered by an air-filled circular cylindrical shell immersed in water", Aerospace Science and Technology. Vol. 27, No. 1, pp. 216-224, 2013.

[34] G. Maze, "Acoustic scattering from submerged cylinders. MIIR Im/Re: Experimental and theoretical study", Journal of the Acoustical Society of America. Vol. 89, No. 6, pp. 2559-2566, 1991.

[35] M. Laaboubi, E.H. Aassif, R. Latif, A. Dliou, G. Maze, D. Décultot, "Application of the reassignment timefrequency method on an acoustic signals backscattered by an air-filled circular cylindrical shell immersed in water", Aerospace Science and Technology. Vol. 27, No. 1, pp. 216-224, 2013.

[36] A. Baillard, J. Chiumia, D. Décultot, G. Maze, A. Klauson, J. Metsaveer, "Surface wave conversion analysis on a lengthwise soldered circular cylindrical shell", Journal of the Acoustical Society of America. Vol. 124, No. 4, pp. 2061-2067, 2008.

[37] K. Pearson, "On lines and planes of closest fit to systems of points in space, Philosophical Magazine". Vol. 2, No. 11, pp. 559-572, 1901.

[38] R. Fisher, W. Mackenzie, "Studies in crop variation. ii. the manurial response of different potato varieties", Journal of Agricultural Science. Vol. 13, No. 3, pp. 311-320, 1923.

[39] H. Hotelling, "Analysis of a complex of statistical variables into principal components", Journal of Educational Psychology. Vol. 24, No. 6, pp. 417-441, 1933.

[40] H. Abdi, L. Williams, "Principal component analysis", Wiley Interdisciplinary Reviews: Computational Statistics. Vol. 2, No. 4, pp. 433-459, 2010.

[41] S. Valle, W. Li, S. Qin, "Selection of the number of principal components: the variance of the reconstruction error criterion with a comparison to other methods", Industrial \& Engineering Chemistry Research. Vol. 38, No. 11, pp. 4389-4401, 1999.

[42] H. Kaiser, "The application of electronic computers to factor analysis", Educational and Psychological Measurement. Vol. 20, No. 1, pp. 141-151, 1960.

[43] R. Cattell, "The scree test for the number of factors", Multivariate Behavioral Research. Vol. 1, No. 2, pp. 245-276, 1966.

[44] M. Negnevitsky, Artificial intelligence: A Guide to Intelligent Systems, 2 ed., Addison-Wesley, New York. 2005.

[45] G. Dreyfus, Neural networks: Methodologie and application, 1 ed., Springer-Verlag Berlin Heidelberg. 2005.

[46] V. Cherkassky, H. Lari-Najafi, "Data representation for diagnostic neural networks", IEEE Expert. Vol. 7, No. 5, pp. 43-53, 1992.

[47] I. Leontaritis, S. Billings, "Model selection and validation methods for nonlinear systems", International Journal of Control. Vol. 45, No. 5, pp. 311-341, 1987. 